Western North American Naturalist 69(3), (C) 2009, pp. 399-402

\title{
REPRODUCTIVE TRAITS IN THE NORTHERN SCORPION (PARUROCTONUS BOREUS)
}

\author{
James N. Barron ${ }^{1,2}$ and Amy L. Weidlich ${ }^{1}$
}

\begin{abstract}
Despite its large geographic range, little is known about reproductive traits in the northern scorpion (Paruroctonus boreus). We analyzed reproductive traits for 36 females from a population near Billings, Montana. All data were collected within a single year. Litter size, offspring mass, total litter mass (TLM), and relative litter mass (RLM) were within the ranges of values reported for other species in the Vaejovidae. Female size (length or mass) was not correlated with any reproductive trait. Litter size and offspring mass were each positively correlated with RLM, suggesting that females investing relatively larger amounts of energy in reproduction increase both size and number of offspring. Finally, the within-litter coefficient of variation in offspring mass was negatively correlated with RLM, TLM, and mean offspring mass, suggesting that females investing more energy in reproduction produce more-uniformly sized offspring, an observation that appears common in scorpions.
\end{abstract}

Key words: litter size, offspring size, relative litter mass.

The northern scorpion Paruroctonus boreus is one of the most widespread scorpions in North America, occurring from southern Canada into Arizona (Fet et al. 2000). Despite its broad geographic range, little is known about reproductive traits in this species. Tourtlotte (1974) provided the only record of litter size in $P$. boreus from 2 females that gave birth in the lab and 1 that died prior to parturition and was dissected. Because of Tourtlotte's small sample size and the lack of data on offspring size, female size, or total litter mass, we initiated a detailed investigation of reproductive traits in $P$. boreus. Our purpose was to add to the growing list of scorpion species for which reproductive data are known.

Scorpions were collected in April and May 2006 near Billings, Montana. Daytime collections were made by turning rocks and surface debris, and nighttime collections were made by using a flashlight with UV-emitting bulbs. Adult female scorpions were collected and housed individually on the campus of Montana State University Billings in plastic containers $(13 \times 9 \times 7 \mathrm{~cm})$ with approximately $1 \mathrm{~cm}$ of sand substrate. Containers were lightly misted once each week, and small crickets (approximately $7.5 \mathrm{~mm}$ long) were offered as food every other week. Most females ate 1 or 2 crickets per month while in captivity. Females were held on a 16L:8D photoperiod until parturition.

Within 24 hours of birth, the live first-instar young were removed from the female's dorsum, counted, and weighed individually to the nearest $0.1 \mathrm{mg}$. Offspring mass was analyzed as the mean of all live offspring for each female. Dead offspring were present in 5 of the 36 litters. Dead offspring represented a relatively small portion of the litter in each case ( 2 of 17,3 of 38, 1 of 24,4 of 23 , and 6 of $25)$ and were not weighed but were included in determination of litter size. Total litter mass (TLM) was calculated as the sum of live offspring mass (for the 31 females that produced only live offspring) or as the product of the mean offspring mass (live) and the total number of offspring (i.e., litter size) for the 5 females that produced some dead young. Female postparturient mass was recorded to the nearest $1.0 \mathrm{mg}$. Female length (prosoma and mesosoma combined) was recorded to the nearest $0.5 \mathrm{~mm}$ (carapace length alone was not recorded). Relative litter mass (RLM) was calculated as the ratio of total litter mass to female postparturient mass. We used an unbiased coefficient of variation $\left(\mathrm{V}^{*}\right.$; Sokal and Rohlf 1995) to assess within-litter variation in offspring mass. All variables were normally distributed (Kolmogorov-Smirnov test, $P>$

${ }^{1}$ Department of Biological and Physical Sciences, Montana State University Billings, 1500 University Drive, Billings, MT 59101

2E-mail: jbarron@msubillings.edu 
Table 1. Descriptive statistics for reproductive traits in Paruroctonus boreus from Montana. Female length is the combined lengths of the prosoma and the mesosoma. Female mass is postparturient mass. $\mathrm{MOM}=$ mean offspring mass, $\mathrm{TLM}=$ total litter mass, and RLM $=$ relative litter mass.

\begin{tabular}{lcccc}
\hline Trait & $n$ & Mean & $s$ & Range \\
\hline Female length $(\mathrm{mm})$ & 36 & 18.7 & 1.4 & $15.5-21.5$ \\
Female mass $(\mathrm{mg})$ & 36 & 470 & 88 & $311-709$ \\
Litter size & 36 & 22.7 & 8.9 & $4-39$ \\
MOM (mg) & 36 & 198.7 & 2.1 & $5.2-12.7$ \\
TLM (mg) & 36 & 0.43 & 0.19 & $26-395$ \\
RLM & 36 & & & $0.07-0.84$ \\
\hline
\end{tabular}

0.20 for each). Correlation analyses (Pearson's $r$ ) were performed, and the minimum significance level was set at $P=0.004$ after sequential Bonferroni correction (Rice 1989) for multiple tests (initial $n=21$ ). Statistical analyses were performed with Statistica for Windows 6.0 (StatSoft, Inc. 2001).

Scorpions gave birth from 25 July to 25 August 2006, though most (30 of 36) gave birth over the 2-week period between 31 July and 14 August. Date of birth was not significantly correlated with any trait. The dates of birth in our study agree closely with that in the only other study on this species (Tourtlotte 1974; 2 females gave birth on 17 August) as well as with those of the congener P. utahensis (Bradley 1984) and the closely related Smeringurus mesaensis (Polis and Farley 1979).

Tourtlotte (1974) is the only other study to report data on female size and litter size in $P$. boreus. Unfortunately, it is unclear whether the females he measured were also those for which he reported litter size. However, the average size of 4 females in his study (prosoma plus mesosoma, calculated from Tourtlotte [1974], $\bar{x}=17.7 \mathrm{~mm}$ ) is similar to the average for the 36 females in our study (Table 1). Tourtlotte reported litter size for 3 females: 2 that gave birth to live young (13 and 39 offspring, respectively) and 1 that was dissected and contained 51 embryos. The range of litter sizes that we found was similar (Table 1).

Mean litter size in our study $(\bar{x}=22.7$, range 4-39; Table 1) was larger than that found for $P$. utahensis (median $=11$, range $2-32$; from Bradley 1984) but smaller than that found for S. mesaensis $(\bar{x}=33$, range 9-53; from Polis and Farley 1979). Thus, based on a fairly limited dataset, it appears that our population of $P$. boreus is intermediate in litter size compared with the other 2 similar species for which litter size has been reported; it is also near the mean litter size (approximately 26) for the order
Scorpiones as a whole (Polis and Sissom 1990). Three of our 36 litters included fewer than 10 offspring (litters of 4, 5, and 8 live neonates) and thus seemed anomalously small. However, because we have no evidence that these litters had been partially cannibalized or were otherwise incomplete, we have included them in all further analyses. Additionally, the range of litter sizes that we found (Table 1) is similar to ranges reported for the related species (above), and removal of the small litters does not qualitatively change the conclusions from the correlation analyses (Table 2) that follow, except as noted.

The average wet mass of first-instar offspring (Table 1) was somewhat intermediate compared with those of other species of Vaejovidae for which offspring mass has been reported. Species with smaller offspring include Vaejovis waueri (Brown and Formanowicz 1996) and Pseudouroctonus apacheanus (Brown 2004), both of which are also significantly smaller as adults than P. boreus. Bradley (1984) reported a mean offspring mass of $8 \mathrm{mg}$ for P. utahensis, and Brown (2004) reported a mean offspring mass of $9.4 \mathrm{mg}$ for Vaejovis spinigerus. Finally, Polis and Farley (1979) reported a range from 29.7 to $39.3 \mathrm{mg}$ for second-instar S. mesaensis. It should be noted, however, that all these offspring sizes were measured on second-instar offspring. Second-instar Centruroides vittatus are approximately $26 \%$ lighter than the first instars (Formanowicz and Shaffer 1993). Applying this percentage to our data still places $P$. boreus in an intermediate position with regards to offspring mass for the Vaejovidae.

Mean total litter mass (TLM; Table 1) and mean relative litter mass (RLM; Table 1) were also intermediate compared to the known masses of other species in Vaejovidae. As would be expected, smaller species such as V. waueri and P. apacheanus show lower TLM (Brown and Formanowicz 1996, Brown 2004) while 
TABLE 2. Correlations among reproductive traits in Paruroctonus boreus from Montana. For all correlations $n=36$. Correlation coefficients (Pearson's $r$ ) are above the diagonal, and $P$ values are below the diagonal. FL $=$ combined prosoma and mesosoma lengths of maternal females, $\mathrm{FM}=$ female postparturient mass, $\mathrm{LS}=$ litter size, $\mathrm{MOM}=$ mean offspring mass, TLM = total litter mass, RLM = relative litter mass, and CVOM = within-litter coefficient of variation for offspring mass. $P$ values that are significant after sequential Bonferroni correction are indicated by an asterisk (*).

\begin{tabular}{lcccccrr}
\hline & FL & FM & LS & MOM & TLM & RLM & \multicolumn{1}{c}{ CVOM } \\
\hline FL & & 0.91 & 0.34 & -0.17 & 0.22 & -0.18 & -0.09 \\
FM & $<0.001^{*}$ & & 0.22 & -0.18 & 0.13 & -0.30 & 0.02 \\
LS & 0.05 & 0.19 & & -0.02 & 0.86 & 0.71 & -0.40 \\
MOM & 0.33 & 0.30 & 0.92 & & 0.47 & 0.55 & -0.62 \\
TLM & 0.21 & 0.45 & $<0.001^{*}$ & $0.004^{*}$ & & 0.90 & -0.59 \\
RLM & 0.30 & 0.08 & $<0.001^{*}$ & $0.001^{*}$ & $<0.001^{*}$ & & -0.61 \\
CVOM & 0.61 & 0.89 & 0.017 & $<0.001^{*}$ & $<0.001^{*}$ & $<0.001^{*}$ & \\
\hline
\end{tabular}

the larger V. spinigerus has a higher TLM (Brown 2004). However, the intent of RLM is to create an index that is somewhat independent of female size; thus, in the studies mentioned above, RLM was lower in P. apacheanus $($ RLM $=0.30)$ but higher in both Vaejovis species (approximately 0.55 for each).

The results of correlation analyses (Table 2) indicate that female length was not significantly correlated with litter size after Bonferroni correction (though the correlation was strong and positive) and not significantly correlated with any other trait except female postparturient mass, which itself was not significantly correlated with any reproductive trait. These results are similar to those for P. utahensis (Bradley 1984), with the exception that Bradley found a negative correlation between female mass and TLM. Additionally, our results are similar to those for V. waueri (Brown and Formanowicz 1996), V. spinigerus, and P. apacheanus (Brown 2004). However, Brown (2001) did find positive relationships between female mass and both litter size and offspring mass in various $V$. waueri datasets, and he also found a positive correlation between female mass and offspring mass in V. spinigerus. What seems clear is that in contrast to many other invertebrates (Roff 1992), scorpions exhibit no general relationships between female size and reproductive traits within species. Further, these correlations vary from year to year, even within individual populations (Brown 2001), suggesting great plasticity in the reproductive system.

Litter size and mean offspring mass were each significantly positively correlated with both TLM and RLM (Table 2), suggesting that females that invest larger amounts of energy (whether absolute or relative) in reproduction increase both size and number of offspring.
This is further supported by significant positive correlations between 2 sets of residuals and each of TLM and RLM. The residuals are those from regressions of litter size on female length, and from offspring mass on female length (of the correlations for litter-size residuals, the smallest correlation coefficient was that with RLM, $r=0.82, P<0.001$; of the correlations for offspring-mass residuals, the smallest correlation coefficient was that with TLM, $r=0.51$, $P=0.001)$. These results are in general agreement with those from other species in the family (Brown and Formanowicz 1996, Brown 2004).

The coefficient of variation $\left(V^{*}\right)$ was significantly negatively correlated with mean offspring mass, TLM, and RLM (Table 2). $V^{*}$ was also negatively correlated with litter size but not significantly so after sequential Bonferroni correction (Table 2). These results indicate that females producing larger offspring and larger TLM and RLM (and to some extent, larger litter sizes as well) do so while producing moreuniformly sized offspring. Again, these results are in general agreement with other reports of species in Vaejovidae (Brown and Formanowicz 1996, Brown 2004), though geographic variation in these relationships has also been noted (Brown and Formanowicz 1995).

Removal of the 3 smallest litters from the correlation analysis did not change conclusions for any variable pair except those involving $V^{*}$ and its correlation with TLM and RLM. In each case, the correlation remained negative but was no longer statistically significant (TLM: $r=-0.29, P=0.10$; RLM: $r=-0.34, P=0.05$ ). The significant negative correlation between $V^{*}$ and mean offspring mass remained essentially unchanged $(r=-0.63, P<0.001)$. Thus, because we have no evidence that the small litters were in some way only partial litters, 
and because the low end of the range of litter sizes we found is similar to those reported for similar species, and because removal of these litters did not greatly affect interpretation of the results, we prefer to base our conclusions on the larger dataset.

In summary, reproductive traits in $P$. boreus are broadly similar to those found in other members of the Vaejovidae. In comparisons among species, larger species tend to have larger offspring (Brown 2001). Compared with members of the family Vaejovidae for which data are available, $P$. boreus is somewhat intermediate in adult size, and the offspring are similarly intermediate in mass. Total litter mass and RLM likewise fall well within the range previously reported for the family. Female size had little influence on reproductive traits, but those females exhibiting larger TLM and RLM produced larger as well as more numerous offspring. Finally, females producing larger offspring produced more-uniformly sized offspring. These correlations in other species, while often variable across populations and within populations across years (Brown and Formanowicz 1995, Brown 2001), suggest that Paruroctonus boreus is similar to other scorpions in that females generally increase reproductive investment by increasing both offspring size and number simultaneously, while producing offspring with lower intralitter variation in mass.

We thank Lael Lindstrand for help with maintenance of scorpions and data collection. Lisa Barton gave us permission to collect on her property, and Ron Stutterheim assisted with collections.

\section{Literature Cited}

BRadley, R.A. 1984. The influence of the quantity of food on fecundity in the desert grassland scorpion
(Paruroctonus utahensis) (Scorpionida, Vaejovidae): an experimental test. Oecologia 62:53-56.

Brown, C.A. 2001. Allometry of offspring size and number in scorpions. Pages 307-315 in V. Fet and P.A. Selden, editors, Scorpions 2001. In Memoriam Gary A. Polis. British Arachnological Society, Burnham Beeches, U.K.

2004. Life histories of four species of scorpion in three families (Buthidae, Diplocentridae, Vaejovidae) from Arizona and New Mexico. Journal of Arachnology 32:193-207.

Brown, C.A., AND D.R. Formanowicz. 1995. Variation in reproductive investment among and within populations of the scorpion Centruroides vittatus. Oecologia 103:140-147.

1996. Reproductive investment in two species of scorpion, Vaejovis waueri (Vaejovidae) and Diplocentrus linda (Diplocentridae), from West Texas. Annals of the American Entomological Society 89:41-46.

Fet, V., W.D. Sissom, G. Lowe, and M. Braunwalder. 2000. Catalog of the scorpions of the world (17581997). New York Entomological Society, New York.

Formanowicz, D.R., AND L.R. ShafFer. 1993. Reproductive investment in the scorpion Centruroides vittatus. Oecologia 94:368-372.

Polis, G.A., AND R.D. FarLey. 1979. Characteristics and environmental determinants of natality, growth and maturity in a natural population of the desert scorpion, Paruroctonus mesaensis (Scorpionida: Vaejovidae). Journal of Zoology, London 187:517-542.

Polis, G.A., AND W.D. Sissom. 1990. Life history. Pages 161-223 in G.A. Polis, editor, The biology of scorpions. Stanford University Press, Stanford, CA.

RICE, W.R. 1989. Analyzing tables of statistical tests. Evolution 43:223-225.

RofF, D.A. 1992. The evolution of life histories. Chapman and Hall, New York.

SOKAL, R.R., AND F.J. ROHLF. 1995. Biometry. 3rd edition. W.H. Freeman, New York.

StatSoft, InC. 2001. STATISTICA for Windows [user's manual]. Tulsa, OK.

Tourtlotte, G.I. 1974. Studies on the biology and ecology of the northern scorpion, Paruroctonus boreus (Girard). Great Basin Naturalist 34:167-179.

Received 4 September 2008 Accepted 12 February 2009 